This item was submitted to Loughborough's Research Repository by the author.

Items in Figshare are protected by copyright, with all rights reserved, unless otherwise indicated.

\title{
Dynamic and tribological study of a planetary gearbox with local nonlinearities
}

PLEASE CITE THE PUBLISHED VERSION

https://doi.org/10.1177/1464419317720602

\section{PUBLISHER}

SAGE (@ Institution of Mechanical Engineers)

VERSION

AM (Accepted Manuscript)

\section{PUBLISHER STATEMENT}

This work is made available according to the conditions of the Creative Commons Attribution-NonCommercialNoDerivatives 4.0 International (CC BY-NC-ND 4.0) licence. Full details of this licence are available at: https://creativecommons.org/licenses/by-nc-nd/4.0/

\section{LICENCE}

CC BY-NC-ND 4.0

\section{REPOSITORY RECORD}

Salagianni, Konstantina, Pantelis Nikolakopoulos, and Stephanos Theodossiades. 2019. "Dynamic and Tribological Study of a Planetary Gearbox with Local Nonlinearities". figshare. https://hdl.handle.net/2134/26493. 


\title{
Dynamic and Tribological Study of a Planetary Gearbox with Local Nonlinearities
}

\author{
Konstantina Salagianni ${ }^{1}$, Pantelis Nikolakopoulos ${ }^{1 *}$ and Stephanos Theodossiades ${ }^{2}$ \\ ${ }^{1}$ Machine Design Laboratory, Department of Mechanical Engineering and Aeronautics, University of Patras, \\ Patras, 26504, Greece \\ ${ }^{2}$ Wolfson School of Mechanical, Electrical and Manufacturing Engineering, Loughborough University, \\ Loughborough, LE11 3TU, UK
}

\begin{abstract}
A planetary gearbox model comprising five spur gears (sun, ring and three planets) and the carrier, has been developed and analyzed. The influence of gear teeth backlash and friction during mixed regime of lubrication have been taken into consideration. Greenwood and Tripp model is employed while viscous friction is calculated analytically using the functions of Evans and Johnson. A combined tribodynamics modeling approach has been implemented and modal analysis is performed in order to predict the coupled mechanism of tribological and dynamic behavior, subjected to backlash and excited at the gear meshing frequency. The software used for the simulations is ADAMS MSc (Student Edition), where the model variables (concerning gear geometry and forcing functions) have been added in a parametric way. The results showed that small variations of the Dynamic Transmission Error (DTE) affect notably the viscous friction through changing the contact load between the engaged teeth pairs. Also, higher values of the Stribeck oil parameter due to higher film thickness or lower surface roughness in the mixed lubrication regime lead to reduction of the boundary friction, whereas a reduction of the total generated friction occurs when increasing the angular velocity of the input gear body (due to higher film thickness and smaller asperity interactions). The above are leading to reduced power loss of the mechanism. Finally, a characterization of the system dynamics is presented using the calculated eigenvalues and eigenmodes of the corresponding linearised system. Potential interactions with the gear meshing frequency of the system are also examined.
\end{abstract}

Keywords: planetary gearbox, teeth backlash, boundary and viscous friction, Dynamic Transmission Error, mixed lubrication regime

\footnotetext{
${ }^{1 *}$ Corresponding author: Pantelis G. Nikolakopoulos, Tel:+30 2610969421, fax:+30 26109972017 , e-mail: pnikolak@mech.upatras.gr
} 


\section{Introduction}

Gears are widely used machine elements in power transmission applications, characterized by high efficiency. However, they can be subjected to severe operating conditions giving rise to aggressive dynamics. Planetary gears are rather compact mechanisms, excellent for transmitting significant power with large speed reductions (or amplifications). Such mechanisms are used in many applications (e.g. wind turbines, aircraft engines, hybrid car transmissions) because of their large bearing capacity, high reliability and long life-span.

In the work of Bartelmus [1] computer simulations revealed that conditions similar to those occurring at resonance may lead to damage of teeth flanks during the service life of a gearbox system. The time varying teeth meshing stiffness and backlash, which influence the dynamic behaviour of the gearbox, are the main excitation parameters in the model of Łuczko [2], who studied the chaotic vibrations in a single stage spur gear transmission. The dynamics of a back-to-back planetary gear, experimental and numerical modal analysis techniques were investigated by Hammami et.al [3]. The gear teeth backlash is considered as one of the main nonlinearities and it may cause oscillations and inaccuracy, leading to poor performance of control systems in many applications [4-6]. Therefore, the dynamic modeling and performance analysis of planetary gear transmissions with backlash have attracted much attention.

Z. M. Sun [7] established a nonlinear dynamic model of a planetary gear transmission considering backlash and mesh stiffness. M. Hamed [8] presented a mathematical model, where the dynamic transmission error was used to analyze the influence of nonlinear oscillations of spur gear pairs with backlash on planetary gear pairs. Q. L. Huang [9] built an optimized mathematical model of a gear transmission system on the basis of a nonlinear purely rotational dynamic model of a multistage closed-form planetary gear, aiming at minimizing the vibration displacement of the low-speed carrier. A lumped parameter nonlinear torsional vibration model of a single-stage planetary set is proposed by Shyyab and Kahraman [10]. It includes all possible power flow configurations, variation of number of planets in any spacing arrangement and planet mesh phasing configuration.

Greenwood and Tripp [11] presented a method to determine boundary friction between two interacting surfaces. Their method assumes Gaussian distribution of the asperities on the solids (meshing teeth surfaces in the case of the examined system). Evans and Johnson [12] reported an analytical experimental expression for viscous friction under elastohydrodynamic conditions, high loads and non-Newtonian shear behaviour. Schulze et al. [13] presented a report regarding the load distribution in planetary gears using MDESIGN software, where it 
was shown that uniform load distribution on gear flank leads to longer life of the mechanism. The software gives complete product information in the early phase of product life cycle (PLC). Mohammadpour et al. [14] presented a tribo-dynamic model for planetary gear sets of Hybrid-Electric-Vehicle configurations. Their model comprises a 6 degree-of-freedom torsional multi-body dynamic system, as well as a tribological contact model in order to evaluate the lubricant film thickness, friction and efficiency of the meshing gear teeth contacts. A model to simulate the dynamic behaviour of a single-stage planetary gear train with helical gears was developed by Kahraman [15]. His three-dimensional dynamic model includes all six rigid body motions of the gears and the carrier of the planetary mechanism. In this paper, a planetary gearbox, which includes five spur gears (sun, ring and three planets) and the carrier, is modeled in ADAMS environment. A combined tribodynamics modelling approach has been implemented and modal analysis is performed in order to predict the coupled mechanism of tribological and dynamic behaviour (subjected by backlash and excited at the gear meshing frequency). The results show that a small variation of the DTE affects notably the viscous friction through changing the contact load between the engaged teeth pair.

\section{The mechanical system}

The studied planetary gearbox comprises the sun (external spur gear), three planets (external spur gears), one carrier (plate) and one ring (internal spur gear). The gearbox stick diagram is presented in figure 1.

The power can be provided either from the sun-gear (input A) or the carrier (input B) or the combination of those two (inputs A \& B). The power is transmitted through the planets and the carrier to the ring-gear, which is the output of the gearbox. In order to maintain kinematic equilibrium, the following equations relating the angular velocity of the bodies $(\omega)$ and the number of gear teeth $(N)$ have to be fulfilled:

$$
\begin{gathered}
\left(N_{r}+N_{s}\right) \cdot \omega_{c}=\omega_{r} \cdot N_{r}+\omega_{s} \cdot N_{s} \\
i_{c}=\frac{\omega_{s}}{\omega_{r}}=\frac{N_{r}}{N_{s}} \\
\omega_{r}=\omega_{s} /\left(\frac{N_{r}}{N_{s}}+1\right) \\
\omega_{p}=\omega_{r} \cdot N_{r} / N_{p}
\end{gathered}
$$

The fundamental meshing frequency is given as 
$f_{m}=\left(N_{s}\right) \cdot\left(\omega_{s}-\omega_{r}\right)=\omega_{r} \cdot N_{r}=\omega_{p} \cdot N_{p=}\left(\frac{N_{s} \times N_{r}}{\left(N_{s}+N_{r}\right)} \times \omega_{s}\right) / 60$

During the operation of the mechanism, friction forces and backlash take-up seem to increase vibration and noise, leading to reduction of gearbox efficiency. Generally, the reactions applied on each gear could be classified as:

- external (e.g. the input torque)

- moment of inertia (because of the angular velocity variation of each gear body)

- internal friction torque (between the gear pairs)

- dynamic transmission error (DTE) induced torque

\section{Teeth Backlash}

Constructional inaccuracy, intentional shape of the gear involute, as well as gear tooth wear are the reasons behind backlash. As the two gears are in mesh, there is a gap between the teeth surfaces in the meshing zone which contributes to non-linear effects in the system vibrations and noise. The DTE is often used as an indicative variable for predicting the system's vibration and noise. The mathematical expression of the DTE, which relates the angular displacement $(\theta)$, and the rate of change $D \dot{T} E$, is presented below:

$$
\begin{aligned}
& D T E_{12}=f a \cdot\left(\theta_{1} \cdot r_{1}-\theta_{2} \cdot r_{2}\right) \\
& D \dot{T} E_{12}=f a \cdot\left(\dot{\theta}_{1} \cdot r_{1}-\dot{\theta}_{2} \cdot r_{2}\right)
\end{aligned}
$$

The system non-linearity because of the backlash can be expressed by piecewise linear equations where DTE is compared each time with the backlash value (B). The result of this comparison defines the value of $f a$ regulatory factor, which nullifies or not the corresponding torque.

When tooth meshing surfaces are in contact, the stiffness and damping forces are applied and their value depends on the type of contact (single or double teeth pairs), according to a specific frequency of alternation, as shown in Fig. 4. So, backlash reset torques for each gear could be presented as in Fig.5:

wheel 1: $\cos \varphi \cdot r_{1} \cdot\left(D T E_{12} \cdot k_{T 12}+D \dot{T} E_{12} \cdot c_{T 12}\right)$

wheel 2: $\cos \varphi \cdot r_{2} \cdot\left(D T E_{12} \cdot k_{T 12}+D \dot{T} E_{12} \cdot c_{T 12}\right)$ 
These forces are applied on the line of action of the gear-pair and that is why $\cos \varphi$ (gear pressure angle) is part of each body's equation. During teeth meshing, hysteretic material damping needs to be included [14]. The damping coefficient for the contact of a single meshing teeth pair can be obtained as $c=0.009 \mathrm{k} / \mathrm{f}_{\mathrm{m}}$, where $\mathrm{f}_{\mathrm{m}}$ is the meshing frequency. In order to obtain the total damping variation during meshing, a similar approach to the above equation is considered.

\section{Gear Teeth Friction}

During the operation of the planetary system, two kinds of friction are expected to be present: boundary $\left(T_{b}\right)$ and viscous $\left(T_{v}\right)$. The total friction is then calculated for each gearpair and it is assigned to each body [14]:

$T_{F R}=T_{b}+T_{v}$

\subsection{Boundary Friction}

Boundary friction forces are developed because of the asperity interaction of the boundary surfaces and are calculated using the Greenwood and Tripp (1970) model [11]. Contact problems between rough surfaces have been studied by many researchers. It is known that in real life scenarios, the surfaces that are in contact are always rough. The first attempt was in 1966 by Greenwood and Williamson [16] for elastic rough surface contacts, assuming that rough surface asperities deform elastically. In reality, if the material's yield strength is exceeded, elasto-plastic deformations occur. The latter scenario was investigated later. The Greenwood and Williamson [16] model assumes Gaussian distribution for asperity summits and the contact of two rough surfaces is considered as that of a rough surface that deforms elastically and a flat surface that is rigid. In the model, the asperities have the same radius for simplicity and the summits follow a Gaussian (height) distribution. Greenwood and Tripp [11] applies the contact model for two rough surfaces.

Continuous efforts of researchers have expanded the study of the basic assumptions of Greenwood and Williamson [11, 16-18] and new methods have been proposed, such as fractal theory, numerical methods etc. [19-20]. In this work the authors are using the expanded model of Greenwood and Williamson extended by Greenwood and Tripp for line contacts. 
According to this model, the friction force for the boundary surfaces $f_{b}$ can be expressed as:

$f_{b}=\tau_{L} \cdot A_{a}$

As it concerns the lubricant shear stress $\tau_{L}$ :

$\tau_{L}=\tau_{O}+\varepsilon \cdot P_{m}$

where $P_{m}=\frac{W_{a}}{A_{a}}$

In order to calculate $A_{a}$ (asperity contact area) and $W_{a}$ (share of the contact load carried by the asperities), the statistical functions $F_{2}, F_{5 / 2}$ are used. These are polynomial functions of the Stribeck oil parameter (see Appendix) to estimate the distribution of asperity heights:

$A_{a}=\pi^{2} \cdot(\xi \cdot \beta \cdot \sigma)^{2} \cdot A \cdot F_{2}(\lambda)$

$W_{a}=\frac{16 \sqrt{2}}{15} \cdot \pi \cdot(\xi \cdot \beta \cdot \sigma)^{2} \cdot \sqrt{\frac{\sigma}{\beta}} \cdot E \cdot A \cdot F_{5 / 2}(\lambda)$

The Stribeck oil parameter $(\lambda)$ is defined as the ratio of the lubricant film thickness (h) to the surface roughness $(\sigma), \lambda=\frac{h}{\sigma} \leq 3$. The different values of Stribeck oil parameter $(\lambda$ less than 3) studied are in the range proposed of references [1, 2], indicating a mixed regime of lubrication close to boundary regime. Below, in Fig. 6, the typical Stribeck curve is presented, relating the friction coefficient to the Stribeck oil parameter. Three regimes of lubrication are present.

Mechanical components operate under lubricated conditions, where the main function of lubricant is the reduction of both friction and wear of the sliding parts.

In most cases, the relationship between friction and lubrication is characterized based on the function $\eta \mathrm{V} / \mathrm{W}$ (oil viscosity $\mathrm{x}$ sliding velocity / normal load, Wakuri et al. [22]) in a curve called Stribeck diagram, reproduced from Bayer [21]. The friction behaviour in the Stribeck diagram is used to explain rubbing phenomena occurring in lubricated contacts. In high values of $\eta U / W$, the friction coefficient is linearly ascending due to fluid film 
lubrication; friction is related to viscous forces in the oil film. When load increases or oil viscosity and/or velocity decreases, the $\eta \mathrm{U} / \mathrm{W}$ factor falls. Then, the fluid film becomes thinner and, consequently, the friction coefficient decreases, up to a minimum value. For even smaller values of $\eta \mathrm{U} / \mathrm{W}$, the fluid film thickness is further reduced, and metal-tometal contact starts to occur. Then, the friction coefficient increases as the $\eta \mathrm{U} / \mathrm{W}$ factor decreases. On the other hand, in the case of two rough surfaces, several authors, such as Hutchings [23]; Bayer [21]; Neale, [24], consider the $\lambda$ value to characterize lubrication in rubbing contacts. This is determined by the relation of oil film thickness (h) and the equivalent surface roughness of both surfaces $(\sigma)$. The oil film thickness $\mathrm{h}$ can be determined from calculations of the elastohydrodynamic film, such as those described in 1960’s by Dowson et al. [25].

$\lambda$ value has been used to analyze wear and friction responses to a great extent in the literature. However it can be considered somewhat inconsistent by Cann et.al [26], because some microscopic effects, such as the micro-elastohydrodynamic lubrication at the asperities, cannot be explained through $\lambda$ value, because the film thickness becomes smaller than the height of surface asperities and then boundary lubrication takes place.

The simplest method to obtain a Stribeck curve and the method most commonly used, provided one has the appropriate converging gap geometry, is to keep two variables fixed (e.g., load and viscosity) and vary the third (e.g., velocity) over a suitable range so that the contact interface goes through the region of asperity contact (boundary), as well as full fluid-film separation (hydrodynamic). In this work the examined mechanism is operating in mixed lubrication regime and the $\lambda$ parameter was chosen to present directly the obtained results with the film thickens variation due to backlash.

\subsection{Viscous Friction}

Viscous friction is a shear force exerted on the teeth surfaces due to the presence of the lubricant film. The analytical experimentally obtained expression of Evans and Johnson (1986) is used, which takes into consideration the influence of generated heat during function [12].

$$
\begin{aligned}
& f_{v}=F_{\text {flank }} \cdot\left(0.87 \cdot a \cdot \tau_{0}+1.74 \cdot \frac{\tau_{0}}{\bar{p}} \cdot \ln \left(\frac{1.2}{\tau_{0} \cdot h} \cdot\left(\frac{2 \cdot \dot{K} \cdot \eta_{0}}{1+9.6 \cdot \zeta}\right)^{1 / 2}\right)\right) \\
& \zeta=\frac{4}{\pi} \cdot \frac{\dot{K}}{h / R(X)} \cdot\left(\frac{\bar{p}}{\dot{E} \cdot R(X) \cdot \dot{K} \cdot \dot{\rho} \cdot c^{\prime} \cdot V}\right)^{1 / 2}
\end{aligned}
$$


Important variables in this calculation are the flank load and the lubricant film thickness.

$$
\begin{aligned}
& F_{\text {flank }}=D T E \cdot k_{T}+D \dot{T} E \cdot C_{T} \\
& h=2.5 \cdot R(X) \cdot \frac{\left(\frac{V(X) \cdot \eta_{0} \cdot \alpha}{R(X)}\right)^{0.7} \cdot(a \cdot \dot{E})^{0.1}}{\left(\frac{F_{\text {flank }} \cdot a}{2 \cdot \mathrm{L} \cdot b}\right)^{0.26}}
\end{aligned}
$$

as per reference [27]

\section{Power Loss}

While boundary and viscous friction forces are applied on each body of a gear-pair, the power loss is the product of the total friction force with the relative sliding velocity between these two gears.

$P=f_{\text {tot }} \cdot \Delta V(X)$

Thus, the friction torque for the gear is given by:

$$
T=\frac{P}{\omega}
$$

\section{Equations of Motion}

For the gears of the planetary system, the following equations of motion are obtained:

Sun: $J_{s} \cdot \ddot{\theta}_{S}+\cos \varphi \cdot r_{s} \cdot \sum_{i=1}^{3}\left(D T E_{s p i} \cdot k_{T s p i}+D \dot{T} E_{s p i} \cdot c_{T s p i}\right)-T_{F R S}=T_{s}$

Planet: $J_{p i} \cdot \ddot{\theta}_{p i}-\cos \varphi \cdot r_{p i} \cdot\left(D T E_{s p i} \cdot k_{T s p i}+D \dot{T} E_{s p i} \cdot c_{T s p i}\right)+\cos \varphi \cdot r_{p i}$.

$\left(D T E_{p r i} \cdot k_{T p r i}+D \dot{T} E_{p r i} \cdot c_{T p r i}\right)-T_{F R p i}=0$

Ring: $J_{r} \cdot \ddot{\theta}_{r}-\cos \varphi \cdot r_{r} \cdot \sum_{i=1}^{3}\left(D T E_{p r i} \cdot k_{T p r i}+D \dot{T} E_{p r i} \cdot c_{T p r i}\right)-T_{F R r}=-T_{r}$

The first term refers to the body's inertia; the second one is the contribution of the DTE; the third term is the total friction torque that is applied on the body; the sum of those three terms is equal to the external torque that is exercised on each gear. For the sun-gear the external torque is the input torque to the gearbox system. As for the ring, this term 
means the resisting torque (the applied load) that is transferred out of the planetary mechanism.

If the carrier is fixed, the DTE equations are as below:

$D T E_{s p}=f a \cdot\left(\theta_{s} \cdot r_{s}-\theta_{p} \cdot r_{p}\right)$

$D T E_{p r}=f a \cdot\left(\theta_{p} \cdot r_{p}-\theta_{r} \cdot r_{r}\right)$

\section{ADAMS Model}

In order to simulate the dynamics of the planetary mechanism, a model has been developed using ADAMS MSC Software (student edition), which allows for dynamic simulations. The constraints and bodies of the model are presented in Table 1:

Table 1: Constrains and bodies of the ADAMS Model

\begin{tabular}{|l|c|c|c|}
\hline \multicolumn{1}{|c|}{ Constrain } & Body 1 & Body 2 & Constrain Point \\
\hline JOINT1/revolute & Ground & Sun & Center mass of sun gear \\
\hline JOINT2/fixed & Ground & Carrier & Carrier center mass \\
\hline JOINT3/revolute & Ground & Ring & Ring center mass \\
\hline JOINT4/revolute & Carrier & Planet 1 & Planet 1 center mass \\
\hline JOINT5/revolute & Carrier & Planet 2 & Planet 2 center mass \\
\hline JOINT6/revolute & Carrier & Planet 3 & Planet 3 center mass \\
\hline
\end{tabular}

The gear forces are calculated analytically with the model variables set in a parametric way. The atmospheric dynamic viscosity of the lubricant is $0.08 \mathrm{~Pa} \cdot \mathrm{s}$, at about $70^{\circ} \mathrm{C}$. The simulations carried out refer to the case that the input of the mechanism is at the sungear, while the carrier remains stationary. The basic parameters of the sun, planets and ring are presented in Table 2.

Table 2: Geometrical features of the studied gearbox

\begin{tabular}{|c|c|c|c|}
\cline { 2 - 4 } \multicolumn{1}{c|}{} & Sun & Planet & Ring \\
\hline Number of teeth & 30 & 20 & 70 \\
\hline Module[mm] & \multicolumn{3}{|c|}{0.8} \\
\hline Pressure angle $\left[{ }^{\circ}\right]$ & \multicolumn{3}{|c|}{21.34} \\
\hline
\end{tabular}

Different values of the backlash (B), Stribeck oil parameter $(\lambda)$ and angular velocity $(\omega)$ in the input body (sun-gear) are leading to interesting case studies. These are the following: 
- For $B[m]: 2.0 e-7,2.5 e-7,3.0 e-7,3.5 e-7$

- For $\lambda[-]: 1,1.25,1.5,1.75,2$

- For $\omega[\mathrm{rpm}]: 1000,2000,3000$.

In Table 3 the basic roughness and lubricant parameters are presented.

Table 3: Lubricant and roughness parameters

\begin{tabular}{|c|c|c|}
\hline Symbol & Value & Units \\
\hline$\xi \beta \sigma$ & 0,055 & - \\
\hline$\sigma / \beta$ & 0,001 & - \\
\hline$\dot{K}$ & 2000 & $\mathrm{~W} / \mathrm{mK}$ \\
\hline$\alpha$ & $2,1 \mathrm{E}-08$ & $1 / \mathrm{Pa}$ \\
\hline$\eta_{o}$ & 0,08 & $\mathrm{~Pa}$ s \\
\hline$\tau_{\mathrm{o}}$ & $2,3 \mathrm{E}+06$ & $\mathrm{~Pa}$ \\
\hline
\end{tabular}

\section{Results and discussion}

In this section, the results for different case studies are displayed, followed by representative plots. It is shown how friction (viscous and boundary) and applied body forces, lubricant film thickness and power losses can differ by changing the values of backlash, Stribeck oil parameter and input angular velocity.

\subsection{Viscous Friction}

As it can be observed from Fig. 7, by increasing the DTE values, the viscous friction between the gear pairs of sun-planet and planet-ring is increasing as well. This can be the result of contact load increase.

\subsection{Boundary Friction}

In Fig. 8, the friction force that is developed as a function of the Stribeck parameter $\lambda$, is presented. In a mixed lubrication regime, higher Stribeck oil parameter, due to higher film thickness or lower surface roughness, leads to reduction of the boundary friction.

\subsection{Applied Body Forces}

The tangential, radial, as well as total applied force and torque at the planet-gear body, are shown in the following plots, when the input torque is about 63Nm. It appears that by increasing the input speed and the $\lambda$ coefficient, the forces are also increasing. 


\subsection{Lubricant Film Thickness}

The diagrams of lubricant film thickness variation for each gear-pair with respect to input body rotation velocity, are presented in Fig. 10. As it can be seen, the lubricant film thickness increases as the rotational speed of the input sun gear increases.

\subsection{Power Losses}

Reduction of the total generated friction seems to occur while increasing the angular velocity of the input gear body (sun). This is caused by higher film thickness and smaller asperity interactions between the engaged teeth pairs. This reduction in friction leads to lower power loss of the mechanism, as shown in Fig.11. The model considers power losses due to friction between the meshing gear teeth and viscous damping of the spinning gear shafts.

\section{Eigenvalue problem}

The high-power-density design of planetary gear sets combined with their kinematic flexibility in achieving different speed ratios makes planetary gear transmissions often an optimum choice. The eigenproblem of the prescribed system (Figure 1) is solved using the corresponding linearised system.

The dynamic models of the sun - planet, ring - planet and carrier - planet are shown in Figures 12a-c. Each gear or carrier is generally allowed to translate in $\mathrm{x}, \mathrm{y}$ and $\mathrm{z}$ (axial) directions and rotate in $\rho_{x}, \rho_{y}$ and $\theta$ directions as per [15]. A displacement vector $\mathbf{q}_{\mathbf{j}}$ and a mass matrix $\mathbf{m}_{\mathbf{j}}$, corresponding to $q$, can be defined for each component $\mathrm{j}$ ( $\mathrm{j}=$ sun, planet, ring, carrier) as below:

$$
\begin{aligned}
& \mathbf{q}_{j}=\operatorname{diag}\left[x_{j}, y_{j}, z_{j}, w_{x j}, w_{y j}, u\right]^{T} \\
& \mathbf{m}_{j}=\operatorname{diag}\left[m_{j}, m_{j}, m_{j}, I_{j} / r_{j}{ }^{2}, I_{j} / r_{j}{ }^{2}, j_{j} / r_{j}{ }^{2}\right]
\end{aligned}
$$

where $m_{j}, I_{j}$ and $J_{j}$ are the mass, the diametral and polar mass moments of inertia, respectively and $w_{x j}=r_{j} \rho_{x j}, w_{y j}=r_{j} \rho_{y j}, u=r_{j} \theta_{j}$.

The undamped equations of motion for the twelve degrees of freedom of the sun - planet pair take the following form: 
$\left[\begin{array}{cc}\mathbf{m}_{s} & 0 \\ 0 & \mathbf{m}_{p_{i}}\end{array}\right]\left\{\begin{array}{l}\ddot{\mathbf{q}}_{s} \\ \ddot{\mathbf{q}}_{p i}\end{array}\right\}+\left[\begin{array}{cc}k_{s i} & -k_{s i} \\ -k_{s i} & k_{s i}\end{array}\right]\left\{\begin{array}{l}\ddot{\mathbf{q}}_{s} \\ \ddot{\mathbf{q}}_{p i}\end{array}\right\}=\left\{\begin{array}{c}\mathbf{f}_{s i} \\ \mathbf{f}_{s p i}\end{array}\right\}$

For the ring - planet it is:

$\left[\begin{array}{cc}\mathbf{m}_{r} & 0 \\ 0 & \mathbf{m}_{p i}\end{array}\right]\left\{\begin{array}{c}\ddot{\mathbf{q}}_{r} \\ \ddot{\mathbf{q}}_{p i}\end{array}\right\}+\left[\begin{array}{cc}k_{r i} & -k_{r i} \\ -k_{r i} & k_{r i}\end{array}\right]\left\{\begin{array}{c}\ddot{\mathbf{q}}_{r} \\ \ddot{\mathbf{q}}_{p i}\end{array}\right\}=\left\{\begin{array}{c}\mathbf{f}_{r i} \\ \mathbf{f}_{r p i}\end{array}\right\}$

While for the planet - carrier it is:

$\left[\begin{array}{cc}\mathbf{m}_{c} & 0 \\ 0 & \mathbf{m}_{p i}\end{array}\right]\left\{\begin{array}{l}\ddot{\mathbf{q}}_{c} \\ \ddot{\mathbf{q}}_{p i}\end{array}\right\}+\left[\begin{array}{cc}k_{c 11 i} & -k_{c 12 i} \\ -k_{c 12 i}^{T} & k_{c 22 i}\end{array}\right]\left\{\begin{array}{l}\ddot{\mathbf{q}}_{c} \\ \ddot{\mathbf{q}}_{p i}\end{array}\right\}=\left\{\begin{array}{c}\mathbf{f}_{c i} \\ \mathbf{f}_{r p i}\end{array}\right\}$

The $K_{s i}$ and $K_{r i}$ and $K_{c 12 i}$ and $K_{c 11 i}$ matrices are given by [15]:

$K_{s i}=K_{s p}\left[\begin{array}{cccccc}c^{2} \beta c^{2} \psi_{s i} & -c^{2} \beta c \psi_{s i} s \psi_{s i} & -c \beta s \beta c \psi_{s i} & c \beta s \beta c^{2} \psi_{s i} & c \beta s \beta c \psi_{s i} s \psi_{s i} & c^{2} \beta c \psi_{s i} \\ & c^{2} \beta s^{2} \psi_{s i} & c \beta s \beta s \psi_{s i} & -c \beta s \beta c \psi_{s i} s \psi_{s i} & c \beta s \beta s^{2} \psi_{s i} & -c^{2} \beta s \psi_{s i} \\ & & s^{2} \beta & -s^{2} \beta c \psi_{s i} & s^{2} \beta s \psi_{s i} & -c \beta s \beta \\ & & & s^{2} \beta c^{2} \psi_{s i} & -s^{2} \beta c \psi_{s i} s \psi_{s i} & c \beta s \beta c \psi_{s i} \\ & & & & s^{2} \beta s^{2} \psi_{s i} & -c \beta s \beta s \psi_{s i} \\ & & & & & c^{2} \beta\end{array}\right]$

(25)

$K_{r i}=K_{s p}\left[\begin{array}{cccccc}c^{2} \beta c^{2} \psi_{r i} & -c^{2} \beta c \psi_{r i} s \psi_{r i} & -c \beta s \beta c \psi_{r i} & c \beta s \beta c^{2} \psi_{r i} & c \beta s \beta c \psi_{r i} s \psi_{r i} & c^{2} \beta c \psi_{r i} \\ & c^{2} \beta s^{2} \psi_{r i} & c \beta s \beta s \psi_{r i} & -c \beta s \beta c \psi_{r i} s \psi_{r i} & c \beta s \beta s^{2} \psi_{r i} & -c^{2} \beta s \psi_{r i} \\ & & s^{2} \beta & -s^{2} \beta c \psi_{r i} & s^{2} \beta s \psi_{r i} & -c \beta s \beta \\ & & & s^{2} \beta c^{2} \psi_{r i} & -s^{2} \beta c \psi_{r i} s \psi_{r i} & c \beta s \beta c \psi_{r i} \\ & & & & s^{2} \beta s^{2} \psi_{r i} & -c \beta s \beta s \psi_{r i} \\ & & & & & c^{2} \beta\end{array}\right]$ 
$K_{c 12 i}=\left[\begin{array}{cccccc}-k_{y y} & 0 & 0 & 0 & 0 & 0 \\ 0 & -k_{x x} & 0 & 0 & 0 & 0 \\ 0 & 0 & -k_{z z} & 0 & 0 & 0 \\ 0 & 0 & -k_{z z} c \psi_{i} & -\gamma k_{w_{y} w_{y}} & 0 & 0 \\ 0 & 0 & k_{z z} s \psi_{i} & 0 & -\gamma k_{w_{x} w_{x}} & 0 \\ k_{y y} c \psi_{i} & -k_{z z} s \psi_{i} & 0 & 0 & 0 & 0\end{array}\right]$

$K_{c 11 i}=\left[\begin{array}{ccccccc}k_{y y} & 0 & 0 & 0 & 0 & -k_{y y} c \psi_{i} \\ & k_{x x} & 0 & 0 & 0 & k_{x x} s \psi_{i} \\ & & k_{z z} & k_{z z} c \psi_{i} & -k_{z z} s \psi_{i} & 0 \\ & & & k_{z z} c^{2} \psi_{i}+k_{w_{y} w_{y}} & -k_{z z} c \psi_{i} s \psi_{i} & 0 \\ & & & & & k_{z z} s^{2} \psi_{i}+k_{w_{x} w_{x}} & 0 \\ & & & & & & k_{x x} s^{2} \psi_{i}+k_{y y} c^{2} \psi_{i}\end{array}\right]$

The helix angle $\beta$ is zero (spur gears) and $\psi_{s i}=\psi_{i}-a_{s}, \psi_{r i}=\psi_{i}-a_{r}$, where $a_{r}, a_{s}$ are the transverse operating pressure gear angles, $s \equiv \sin \beta, \quad c \equiv \cos \beta$, $k_{22 i}=\operatorname{diag}\left[k_{x x}, k_{y y}, k_{z z}, \gamma^{2} k_{w_{y} w_{y}}, \gamma^{2} k_{w_{x} w_{x}}, 0\right], \quad \gamma=r_{c} / r_{p}, \quad k_{w_{y} w_{y}}=\rho_{y} \rho_{y} / r_{c}^{2} \quad$ and $k_{w_{x} w_{x}}=\rho_{x} \rho_{x} / r_{c}^{2}$. The angular position of the planets has been defined as $90^{\circ},-30^{\circ}$ and $210^{0}$.

In this work only the rotational (torsional) degree of freedom $\left(u=r_{j} \theta_{j}\right)$ is considered. Thus, the equations of motion (22) - (24) are reduced to the following (for free vibrations):

$\left[\begin{array}{cc}j_{s} & 0 \\ 0 & j_{p i}\end{array}\right]\left\{\begin{array}{c}\ddot{u}_{s} \\ \ddot{u}_{p i}\end{array}\right\}+k_{s p}\left[\begin{array}{cc}c^{2} \beta & -c^{2} \beta \\ -c^{2} \beta & c^{2} \beta\end{array}\right]\left\{\begin{array}{l}u_{s} \\ u_{p i}\end{array}\right\}=\left\{\begin{array}{l}0 \\ 0\end{array}\right\}$

$\left[\begin{array}{cc}j_{r} & 0 \\ 0 & j_{p i}\end{array}\right]\left\{\begin{array}{c}\ddot{u}_{r} \\ \ddot{u}_{p i}\end{array}\right\}+k_{r p}\left[\begin{array}{cc}c^{2} \beta & -c^{2} \beta \\ -c^{2} \beta & c^{2} \beta\end{array}\right]\left\{\begin{array}{l}u_{r} \\ u_{p i}\end{array}\right\}=\left\{\begin{array}{l}0 \\ 0\end{array}\right\}$

$\left[\begin{array}{cc}j_{c} & 0 \\ 0 & j_{p i}\end{array}\right]\left\{\begin{array}{l}\ddot{u}_{c} \\ \ddot{u}_{p i}\end{array}\right\}+\left[\begin{array}{cc}k_{x x} s^{2} \psi_{i}+k y y c^{2} \psi_{i} & 0 \\ 0 & 0\end{array}\right]\left\{\begin{array}{c}u_{c} \\ u_{p i}\end{array}\right\}=\left\{\begin{array}{l}0 \\ 0\end{array}\right\}$ 
The overall planetary system equations of motion can be written as:

$\mathbf{M} \ddot{\mathbf{x}}+\mathbf{K x}=\mathbf{0}$

Where for the rotational mode of vibration,

$\mathbf{M}=\operatorname{diag}\left[J_{s} / r_{s}^{2}, J_{p 1} / r_{p 1}^{2}, J_{p 2} / r_{p 2}^{2}, J_{p 3} / r_{p 3}^{2}, J_{r} / r_{r}^{2}, J_{c} / r_{c}^{2}\right]$

$\mathbf{X}=\left[\theta_{s}, \theta_{p 1}, \theta_{p 2}, \theta_{p 3}, \theta_{r}, \theta_{c}\right]^{T}$

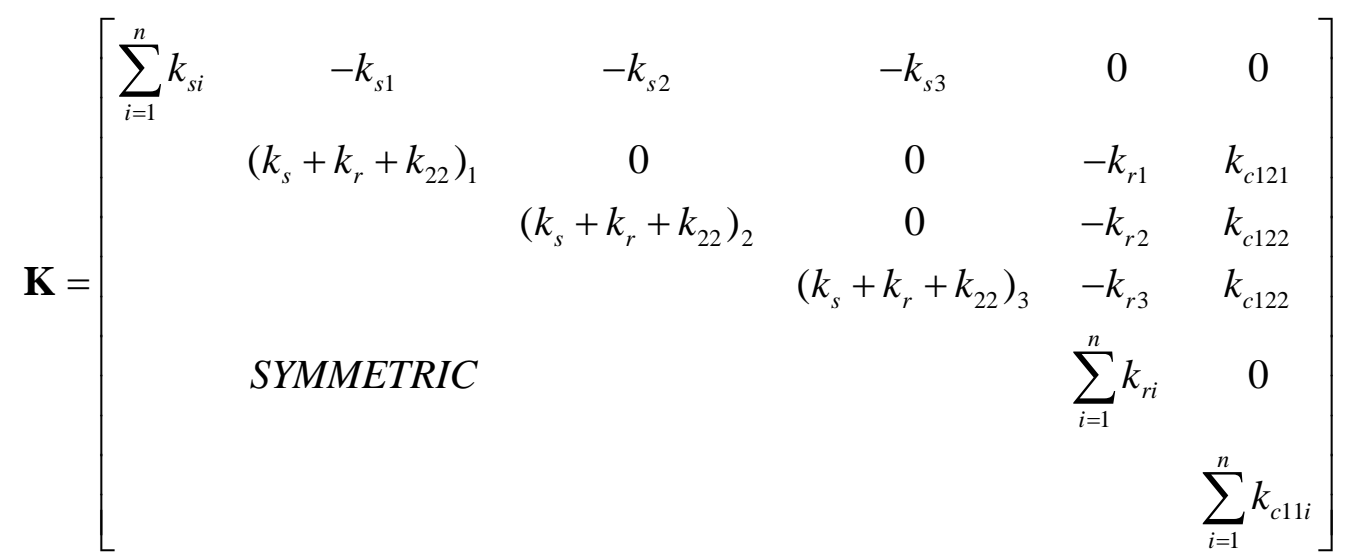

For the time invariant case related the eigenvalue problem can be written as follows, having neglected the gyroscopic terms due to the relatively low carrier rotational speed:

$\left(\mathbf{K}-\mathbf{M} \omega_{i}^{2}\right) \Phi_{i}$

where $\omega_{i}$ are the natural frequencies and $\Phi_{i}$ are the eigenvectors. The system properties of Table 4 are used in order to solve the eigenproblem.

Table 4. Properties of the examined planetary gear system

\begin{tabular}{|c|c|c|c|c|}
\hline & Sun & Ring & Carrier & Planet \\
\hline Mass (kg) & 0.272 & 0.779 & 1.5 & 0.1 \\
\hline $\mathbf{j} / \mathbf{r}^{\mathbf{2}} \mathbf{( k g )}$ & 0.136 & 0.389 & 0.75 & 0.05 \\
\hline Base Diameter (m) & 0.024 & 0.056 & 0.040 & 0.016 \\
\hline
\end{tabular}




\begin{tabular}{|c|c|c|c|c|}
\hline $\begin{array}{c}\text { Mesh single contact } \\
\text { stiffness (N/m) }\end{array}$ & $2.0 \mathrm{E}+08$ & $2.0 \mathrm{E}+08$ & $2.0 \mathrm{E}+08$ & $2.0 \mathrm{E}+08$ \\
\hline
\end{tabular}

The following overall stiffness and mass matrices containing the torsional degrees of freedom have occurred:

$$
\begin{aligned}
& \mathbf{M}=\left[\begin{array}{llllll}
0,272 & & & & & \\
& 0,1 & & & & \\
& & 0,1 & & & \\
& & & 0,1 & & \\
& & & & 0,759 & \\
& & & & & 1,5
\end{array}\right] \\
& K=\left[\begin{array}{cccccc}
6 \mathrm{e}+8 & -2 e+8 & -2 e+8 & -2 e+8 & 0 & 0 \\
& 4 e+8 & 0 & 0 & -2 e+8 & 0 \\
& & 4 e+8 & 0 & -2 e+8 & 0 \\
& & & 4 e+8 & -2 e+8 & 0 \\
& & & & 6 e+8 & 0 \\
& & & & & 8,04 e+8
\end{array}\right]
\end{aligned}
$$

Solving the eigenproblem, the natural frequencies below are obtained for the planetary system (table 5):

Table 5. Natural frequencies of the planetary system for Ksp=2e8N/m

\begin{tabular}{|c|}
\hline fn (Hz) \\
\hline 0 (rigid body mode) \\
\hline 3687 \\
\hline 5956 \\
\hline 10070 \\
\hline 10070 \\
\hline 11948 \\
\hline
\end{tabular}

\subsection{Gear meshing frequency (GMF) effects}

The gear meshing frequency defined as the rotational velocity of the gear wheel multiplied by the number of gear teeth is one of the main noise features in transmissions. Gear meshing creates oscillations in its meshing frequency because of the transmission error, machining errors, stiffness variation, torque fluctuations etc. Consequently, the 
dynamic tooth mesh forces are periodic at the mesh frequency and the calculated response contains integer multiples of the harmonics of the fundamental tooth mesh frequency for the operating speeds considered. This result is consistent with the static transmission error excitation model used in lumped-parameter representations. In planetary gear mechanisms, due to the complicated mechanical structure of power distribution, the gears that transmit power for long periods of time are more susceptible to accumulating tooth wear, resulting in larger transmission errors, reduction of the meshing stiffness, and increase of backlash.

In order to study the modal behaviour of the planetary gear system (table 1 data) under typical operating conditions, the sun speed was taken as 1000rpm and the input torque constant at $63 \mathrm{Nm}$. The meshing frequency is then $f_{m}=350 \mathrm{~Hz}$. During the planetary gear mechanism operation, the mesh stiffness can be mistuned due to manufacturing irregularities. In Figure 13, the variation of the first three natural frequencies of the system is presented. As it can be seen there can be interactions with the meshing frequency that affect the mechanism operation (leading to potential resonances) as Ksp is varied between $1.5 \mathrm{E} 3 \mathrm{~N} / \mathrm{m}$ to $1.5 \mathrm{E} 4 \mathrm{~N} / \mathrm{m}$.

As it can be seen in Figure 14, the vibration modes can change drastically as Ksp varies. Thus, the above analysis can provide planetary system designers with a tool that calculates accurately the dynamic and tribological interactions between the mating gear wheels in the planetary mechanism. It may also assist the proper selection of the gear design parameters to avoid resonance conditions if errors lead to excitation of the mechanism with frequencies close to the fundamental gear meshing frequency and its harmonics.

\section{Conclusions}

In this work, a combined tribodynamics modeling approach has been implemented and modal analysis is performed in order to predict the coupled mechanism of tribological and dynamic behavior of a five-spur gear planetary gearbox. Backlash and excitation at the gear meshing frequency have been considered. The tribological and dynamic characteristics of the system have been investigated.

The results showed that:

- A small variation of DTE affects notably the viscous friction

- Higher Stribeck oil parameter leads to reduction of the boundary friction in the mixed lubrication regime 
- Reduction of the total generated friction seems to occur while increasing the angular velocity of the input gear body. This reduction leads to lower power loss of the mechanism.

- Modal analysis is also performed, showing the effects of the meshing frequency on the eigenvectors and the eigenmodes of the mechanism.

The future work should comprise the addition of all the dynamic parameters and degrees of freedom to the numerical model. Since real word applications require higher efficiencies, an extension of the work could be also to improve the model in terms of choosing interactively the input either from the sun, the carrier or their combination. The time variant properties of the system (transmission error and meshing stiffness) and their effects in the gear box response and power losses will be examined in a future investigation.

\section{Notation}

\begin{tabular}{|c|c|c|c|}
\hline$A$ & apparent contact area & $T$ & torque \\
\hline$A_{a}$ & asperity contact area & $T_{b}$ & boundary friction torque \\
\hline$b$ & half-width of Hertzian contact & $T_{F R}$ & total friction torque \\
\hline$B$ & backlash & $T_{v}$ & viscous friction torque \\
\hline$c$ & damping coefficient & $\tau_{L}$ & lubricant limiting shear stress \\
\hline$\dot{c}$ & $\begin{array}{l}\text { thermal capacity of conjunctional } \\
\text { solids }\end{array}$ & \multirow[t]{2}{*}{$\tau_{O}$} & \multirow{2}{*}{$\begin{array}{l}\text { lubricant's limiting shear stress at } \\
\text { atmospheric pressure }\end{array}$} \\
\hline DTE & dynamic transmission error & & \\
\hline$D \dot{T} E$ & variation of DTE & $V(X)$ & instant velocity \\
\hline$E^{\prime}$ & equivalent modulus of elasticity & 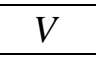 & speed of entraining motion of the lubricant \\
\hline fa & regulatory factor & $W_{a}$ & $\begin{array}{l}\text { share of the contact load carried by the } \\
\text { asperities }\end{array}$ \\
\hline$f_{b}$ & boundary friction force & $X$ & instantaneous meshing position \\
\hline$f_{v}$ & viscous friction force & $\alpha$ & pressure-viscosity coefficient \\
\hline$F_{2}$ & statistical function & \multirow[b]{2}{*}{$\varepsilon$} & \multirow{2}{*}{$\begin{array}{l}\text { pressure-induced shear coefficient of } \\
\text { bounding surfaces }\end{array}$} \\
\hline$F_{5 / 2}$ & statistical function & & \\
\hline$F_{\text {flank }}$ & flank load & $\zeta$ & viscous friction force coefficient \\
\hline$h$ & lubricant film thickness & $\eta_{o}$ & $\begin{array}{l}\text { atmospheric dynamic viscosity of the } \\
\text { lubricant }\end{array}$ \\
\hline$J, I$ & moment of inertia & $\theta$ & angular displacement \\
\hline$k$ & stiffness & $\dot{\theta}$ & angular velocity \\
\hline$k_{\text {in }}$ & stiffness mean values & $\ddot{\theta}$ & angular acceleration \\
\hline$\dot{K}$ & lubricant conductivity & $\lambda$ & Stribeck oil parameter \\
\hline$\dot{K}$ & surface solid conductivity & $\dot{\rho}$ & density of conjunctional solids' material \\
\hline$L$ & gear flank width & $\varphi$ & pressure angle \\
\hline$P$ & power & $\Phi$ & $\begin{array}{c}\text { eigenmodes (in eigenvalue problem } \\
\text { section) }\end{array}$ \\
\hline$P_{m}$ & stress from Wa load at Aa area & $\omega$ & angular velocity \\
\hline $\bar{p}$ & average contact pressure & $\omega$ & eigenfrequencies (in eigenvalue problem \\
\hline
\end{tabular}




\begin{tabular}{|c|c|c|c|}
\hline & & & section) \\
\hline$r$ & gear wheel radius & $\xi \beta \sigma$ & roughness parameter \\
\hline$R(X)$ & instant position & $\sigma / \beta$ & $\begin{array}{c}\text { representation of the average asperity } \\
\text { slope }\end{array}$ \\
\hline $\mathbf{m}$ & Mass matrix & $\mathbf{q j}$ & \begin{tabular}{c} 
Displacements vector \\
\hline $\boldsymbol{f}_{\boldsymbol{m}}$
\end{tabular} \\
\hline
\end{tabular}

\section{Captions list}

\begin{tabular}{|c|c|}
\hline Figure 1: & Gearbox stick diagram \\
\hline Figure 2: & Gear-pair backlash \\
\hline Figure 3: & Values of the fa coefficient \\
\hline Figure 4: & Meshing cycle of the spur gear pair [14] \\
\hline Figure 5: & Gear pair contact model \\
\hline Figure 6: & Stribeck curve \\
\hline Figure 7: & $\begin{array}{l}\text { Viscous friction force vs DTE at 1000rpm input speed for a) sun-planet gear pair, b) } \\
\text { planet-ring gear pair }\end{array}$ \\
\hline Figure 8: & $\begin{array}{l}\text { Boundary friction force vs Stribeck oil parameter for a) sun-planet gear pair, b) planet- } \\
\text { ring gear pair }\end{array}$ \\
\hline Figure 9: & $\begin{array}{l}\text { Applied forces-torque at planet-gear wheel (tangential, radial \& total force and total } \\
\text { torque) vs input speed and Stribeck oil parameter }\end{array}$ \\
\hline Figure 10: & $\begin{array}{l}\text { Lubricant film thickness vs input speed for a) sun-planet gear pair, b) planet-ring gear } \\
\text { pair }\end{array}$ \\
\hline Figure 11: & Power loss ratio vs Stribeck oil parameter and input speed \\
\hline Figure 12. & Degrees of freedom for: a) Sun - Planet, b) Ring - Planet and c) Planet - Carrier \\
\hline Figure 13. & $\begin{array}{l}\text { Variations of the sun, planet } 1 \text { and ring eigenfrequencies as a function of the Ksp } \\
\text { stiffness variation }\end{array}$ \\
\hline Figure 14. & $\begin{array}{l}\text { Qualitative planetatery gear box eigen modes for } \mathrm{Ksp}=1,5 \mathrm{E} 4 \mathrm{~N} / \mathrm{m} \text { and } \\
\mathrm{Ksp}=1,4 \mathrm{E} 3 \mathrm{~N} / \mathrm{m} \text { for sun eigenfrequencies } 8643 \mathrm{~Hz} \text { and } 23 \mathrm{~Hz} \text { respectively }\end{array}$ \\
\hline Table 1: & Constrains and bodies of ADAMS Model \\
\hline Table 2: & Geometrical features of the studied gearbox \\
\hline Table 3: & Lubricant and roughness parameters \\
\hline Table 4: & Properties of planetary gear system \\
\hline Table 5: & Natural frequencies of the planetary system for Ksp=2e8N/m \\
\hline
\end{tabular}

\section{Lengthy mathematical expressions}

$$
\begin{aligned}
& F_{5 / 2}(\lambda)=\left\{\begin{array}{r}
-0.004 \cdot \lambda^{5}+0.057 \cdot \lambda^{4}-0.296 \cdot \lambda^{3}+0.784 \cdot \lambda^{2}-1.078 \cdot \lambda+0.617, \quad \lambda \leq 3 \\
0, \quad \lambda>3
\end{array}\right. \\
& F_{2}(\lambda)=\left\{\begin{array}{r}
-0.002 \cdot \lambda^{5}+0.028 \cdot \lambda^{4}-0.173 \cdot \lambda^{3}+0.526 \cdot \lambda^{2}-0.804 \cdot \lambda+0.5, \quad \lambda \leq 3 \\
0, \quad \lambda>3
\end{array}\right.
\end{aligned}
$$

\section{References}

1. Bartelmus W., Gearbox dynamic modelling, Journal of Theoretical and Applied Mechanics, 2001, 39, 4, 989-999. 
2. Łuczko J.,Chaotic vibrations in gear mesh systems, Journal of Theoretical and Applied Mechanics,2008, 46, 4, 879-896.

3. Ahmed Hammami, Alfonso Fernandez Del Rincon, Fernando Viadero Rueda, Fakher Chaari, Mohamed Haddar, Modal analysis of back-to-back planetary gear: Experiments and correlation against Lumped-parameter model, Journal of theoretical and applied mechanics, 2015, 53, 1, pp. 125-138, Warsaw 2015,DOI: 10.15632/jtam-pl.53.1.125.

4. Tasora A., Prati E.and Silvestri M., A Compliant Measuring System for Revolute Joints with Clearance, Proceedings of the 2006 International Conference on Tribology, 2006, September 20-22; Parma, Italy.

5. Tian Q., Zhang Y. Q. and Dynamics L. P. of Spatial Flexible Multibody Systems with Clearance and Lubricated Spherical Joints, Computers \& StructuresComputers and Structures, 2009,vol. 87, no. 11, pp. 913-929.

6. Erkaya S. and Uzmay I., Determining link parameters using genetic algorithm in mechanisms with joint clearance,Mechanism and Machine Theory, 2009,vol. 44, no. 1, pp. 222-234.

7. Sun Z. M., Ji L. H. and Shen Y. W., Nonlinear dynamics of 2K-H planetary gear train,Journal of Tsinghua University (Science and Technology), 2003,vol. 43, no.5, pp. 636-639.

8. Moradi H. and Salarieh H., Analysis of nonlinear oscillations in spur gear pairs with approximated modelling of backlash nonlinearity, Mechanism and Machine Theory, 2012,vol. 51, no. 5, pp. 14-31.

9. Huang Q., Wang Y.and Huo Z., Nonlinear dynamic analysis and optimization of closed - form planetary gear system, Mathematical Problems in Engineering, vol. 2013, (2013), pp. 1-12.

10. Al-Shyyab A., Kahraman A., A non-linear dynamic model for planetary gear sets, Proceedings of the Institution of Mechanical Engineers, Part K: Journal of Multi-body Dynamics December 1, 2007 vol. 221 no. 4 567-576.

11. Greenwood, J. A. and Tripp, J. H. The contact of two nominally flat rough surfaces, Proc. Instn. Mech. Engrs, 1970-71. 185, pp. 625-633

12. Evans, C. R. and Johnson, K. L. Regimes of traction in elastohydrodynamic lubrication, Proc. Instn. Mech. Engrs., 1986, 200 (C5), pp. 313-324

13. Venkatesh B., Kamala V., . Prasad Me. A.M. K, Design, modeling \& manufacturing of Helical Gear International, Journal of applied Engineering Research Dingidul, Vol-1, 2010, pp-103-114 
14. Mohammadpour M., Theodossiades S.and Rahnejat H.,Dynamics and efficiency of planetary gear sets for hybrid powertrains, Proceedings of the Institution of Mechanical Engineers, Part C: Journal of Mechanical Engineering Science,2015,230(7-8),1359-1368. 15. Kahraman A., Planetary Gear Train Dynamics , Journal of Mechanical Design,ASME, 1994, Vol.116 , 713-720.

16. Greenwood, J. A., and Williamson, J. B. P., Contact of Nominally Flat Surfaces, Proc. R. Soc. London, 1966, Ser. A, 295, pp. 300-319. 3.

17.Greenwood, J.A. and Tripp, J.H., The Elastic contact of Rough Spheres,Apll. Mech, 1967, Vol. 185, 349(1),pp. 153-159.

18.Greenwood, J.A,A simplified Elliptical Model for Rough Surfaces Contact. Wear 261(2):191-200.

19. Majumdar A.and Bhushan B., Fractal Model of Elastic-Plastic Contact Between Rough Surfaces, J. Tribol 1991.113(1), pp.1-11

20.. Paggi M, Ciavarella M., The coefficient of proportionality $\kappa$ between real contact area and load, with new asperity models , Wear, 2010268, 7-8, 9 , pp. 1020-1029.

21. Bayer, R.G., 1994, Mechanical Wear Prediction and Prevention, Marcel Dekker, USA, 657 pp

22. Wakuri, Y., Soejima, M., Kitahara, T., Fujisaki, K., and Nuki, K., , Effect of lubricating oils on piston ring friction and scuffing, Japanese Journal of Tribology, 1995,Vol.40, No.5, pp. 437-449.

23. Dowson, D., “Elastohydrodynamic and microelastohydrodynamic lubrication”, Wear, 1995,190, pp. 125-138

24. Neale,M.J., The Tribology Handbook”, 2nd Ed., USA, Butterworth-Heinemann,1997 25. Hutchings, I.M., Tribology: friction and wear of engineering materials, Edward Arnold, Great Britain, 1992, pp273.

26. Cann, P., Ioannides, E., Jacobson, B., and Lubrecht, A.A., , The lambda ratio - a critical re-examination, ,Wear, 1994,Vol.175, pp. 177-188

27. Dowson, D. and Higginson, G.R., "Elasto-hydrodynamic lubrication”, Pergamon Press, Oxford, 1977 